\title{
Relationship between finger and wrist deformities in rheumatoid arthritis
}

\author{
G. O. READ, L. SOLOMON, AND S. BIDDULPH
}

From the Department of Orthopaedic Surgery, University of the Witwatersrand, Johannesburg, South Africa

SUMmARY The present study was undertaken to test the hypothesis that there is a significant correlation between the position of the wrist and metacarpophalangeal angulation in the common rheumatoid hand deformities. Measurements were carried out on radiographs of the hand from 2 comparative groups: 67 patients (134 hands) with classical or definite rheumatoid arthritis (RA), and a control series of 50 adults (100 hands) attending the routine fracture clinic. Rheumatoid patients were unselected and included those with a variety of hand deformities. The results showed a statistically significant relationship between finger and wrist deformities in patients with RA but not in the normal controls.

Distortion of the metacarpophalangeal joints is the hallmark of rheumatoid disease. Though the commonest deformity is ulnar deviation of the fingers, all manner of joint displacement may occur. Furthermore, symmetrical ulnar drift is by no means exclusive to rheumatoid arthritis, for it is seen also in polyarticular osteoarthritis and even in normal persons, especially with advancing age.

The postural abnormalities of the rheumatoid hand are still largely unexplained. To label the cause as 'multifactorial' may diminish the importance of some underlying mechanical principle which, though it might not explain the occurrence of metacarpophalangeal deformity, could determine its particular direction. Recognition of any such consistent pattern could open the door to effective prophylaxis and corrective treatment.

During the last decade several studies have emphasised the role of the wrist in the pathogenesis of ulnar deviation of the fingers. ${ }^{1-3}$ Severe ulnar drift of the fingers is almost always associated with a counterpoised radial deviation at the wrist (Fig. 1). Whether this is sequential or coincidental remains to be shown. Our aim in the present study was to test the hypothesis that there is a significant correlation between the position of the wrist and metacar-

Accepted for publication 18 November 1982.

Correspondence to Professor L. Solomon, Department of Orthopaedic Surgery, Medical School, York Road, Johannesburg 2001, South Africa. pophalangeal angulation in all rheumatoid hand deformities.

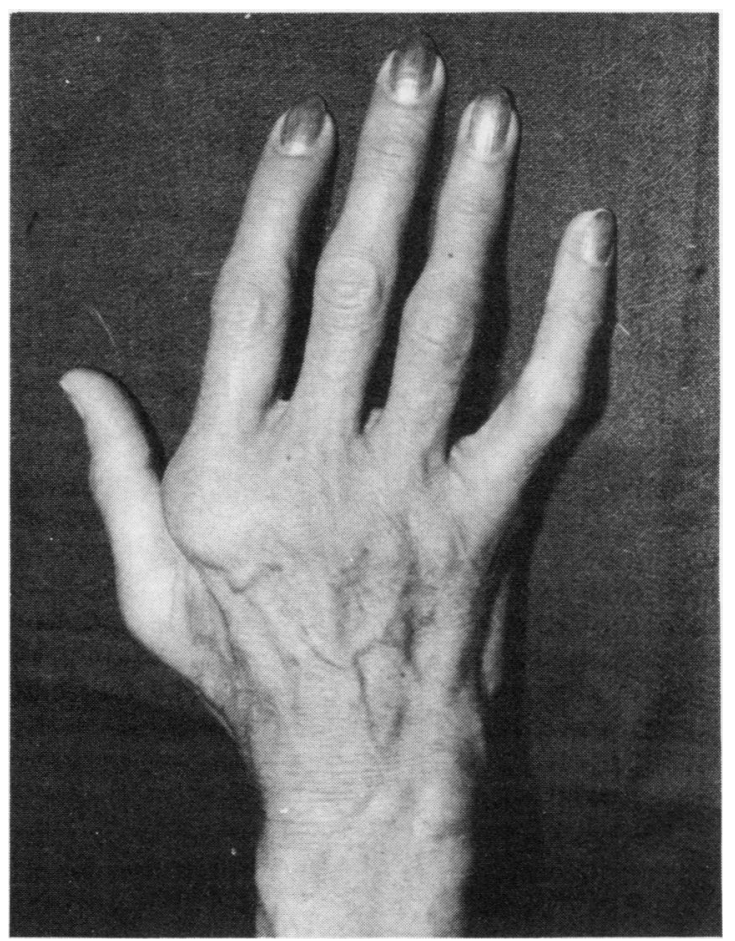

Fig. 1 The typical rheumatoid deformity: ulnar drift of the fingers and radial deviation of the wrist and metacarpus. 


\section{Material and methods}

Two groups were studied: $(a)$ the 'rheumatoid group'-134 hands in 67 consecutive patients with 'classical' or 'definite' rheumatoid arthritis of varying severity and duration; and (b) the 'control group'-100 hands in 50 consecutive adults attending the Fracture Clinic, but excluding those with upper limb injuries.

Anteroposterior radiographs of the hands of all the patients were taken by a standardised technique and by the same radiographer. The hands were positioned palms downward with the forearms supported, if necessary, to ensure that the carpus was flat on the $x$-ray cassette.

The following measurements were carried out on each radiograph:

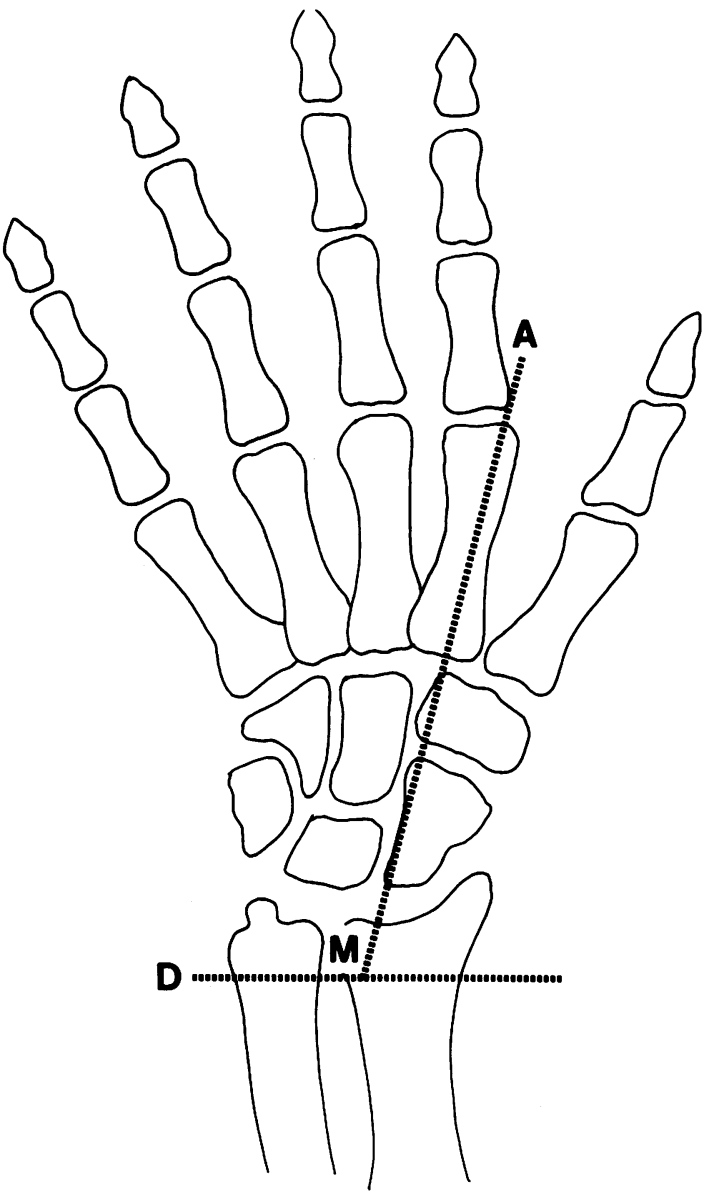

Fig. 2 Diagram showing the co-ordinates of the angle of metacarpal deviation (AMD).
The angle of metacarpal deviation (AMD). The co-ordinates of this angle are a line at right-angles to the shaft of the radius and another along the axis of the second metacarpal (Fig. 2). With a wrist in radial deviation this will be an obtuse angle; in ulnar deviation it will be an acute angle.

The angle of phalangeal drift (APD). This is the angle subtended by lines drawn along the shafts of the second metacarpal and the proximal phalanx of the second finger (Fig. 3).

The ratio of carpal height to radial width (Carpal ratio). Carpal height was taken as the distance from the middle of the base of the third metacarpal to a point on the radius opposite the scapholunate articulation; radial width was measured from the tip of the radial styloid to the ulnar border of the distal end

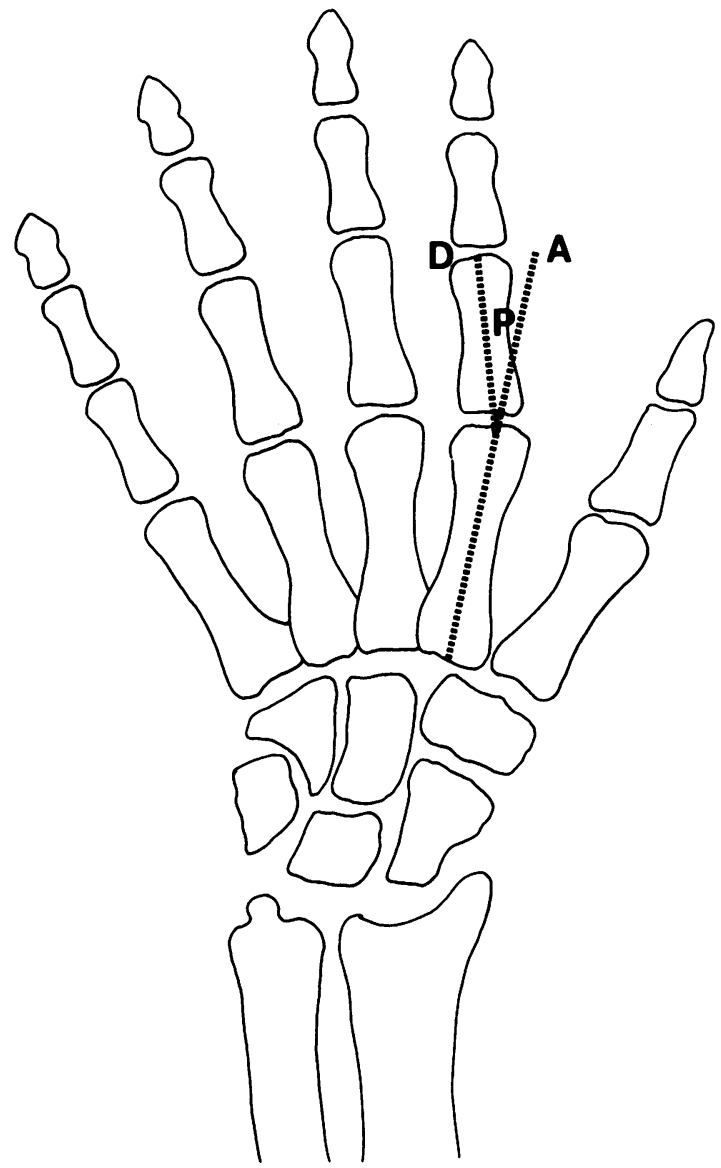

Fig. 3 The angle of phalangeal drift (APD). 


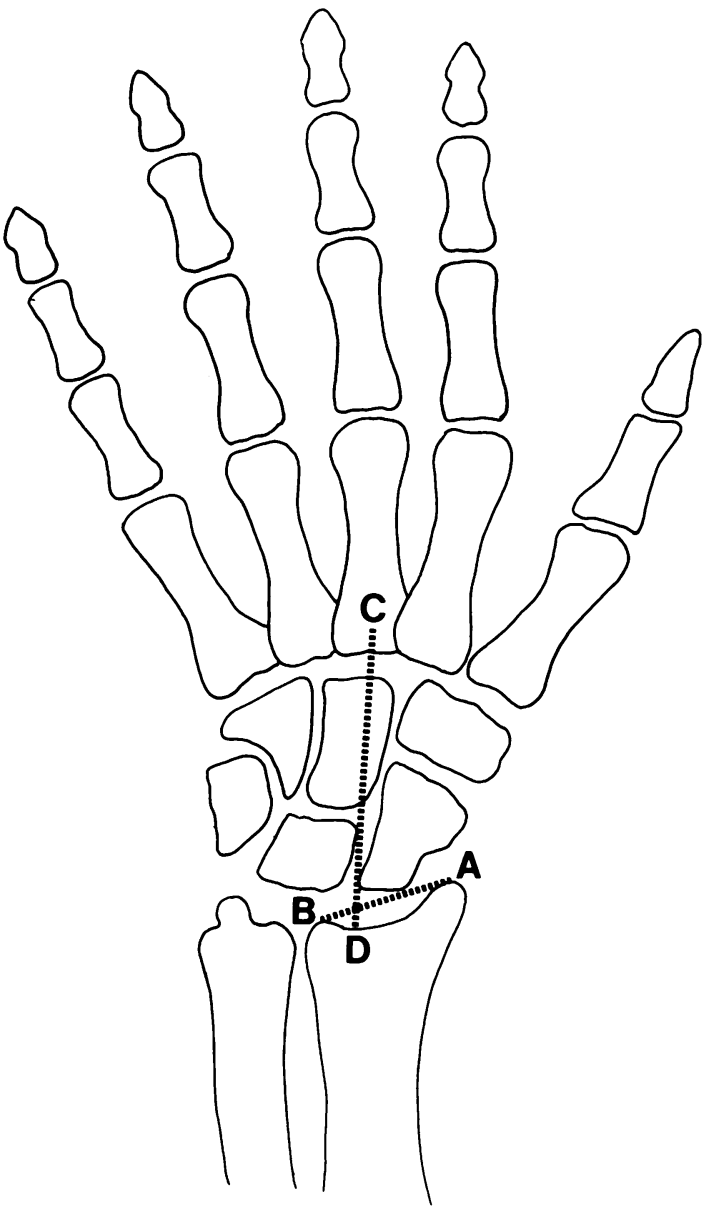

Fig. 4 Measurements used in expressing the 'carpal ratio': $C D / A B$.

of the radius (Fig. 4). The smaller the carpal ratio the greater the degree of carpal collapse.

\section{Results}

Sex and age distribution. $71 \%$ of the rheumatoid group and $53 \%$ of the control group were women. The average age of the former was $53 \cdot 1$ years and of the latter $\mathbf{4 0 . 6}$ years. Although the age and sex distribution was significantly different in the 2 groups, no age or sex times group interaction existed with reference to the variables AMD, APD, and carpal ratio. The control group was thus statistically valid.

Ulnar drift of the fingers. Surprisingly, there was no statistically significant difference in the mean APD between rheumatoid patients and normal persons
(Table 1). This is explained by the fact that $21 \%$ of the rheumatoid group had a negative APD-i.e., the fingers were deviated radialwards at the metacarpophalangeal joints. However, the frequency distribution (Fig. 5) shows that the rheumatoid group also includes a significant number of hands with ulnar drift more marked than anything seen in the normal hand.

Angle of metacarpal deviation. The frequency distribution of the AMD in the 2 groups is shown in Fig. 6 . There was generally a greater degree of radial deviation of the wrist and metacarpals in the rheumatoid group than in the normal persons. This difference was statistically significant $(p<0 \cdot 01)$ for the right hand and $(p<0.05)$ for the left hand (Table 1).

Relationship of ulnar drift to wrist deviation. There was a highly significant correlation between the angle of phalangeal deviation and the position of the wrist in rheumatoid hands $(p<0.01)$ (Table 2$)$. The greater the degree of radial deviation at the wrist the more severe was the ulnar deviation of the fingers (Fig. 7). The opposite was equally true: ulnar deviation of the wrist and metacarpals was associated with the contrary deformity (radial drift) of the fingers (Fig. 8).

Table 1 Results of $\mathrm{t}$ tests comparing rheumatoid patients and normal person within variables

\begin{tabular}{|c|c|c|c|c|}
\hline \multirow[t]{3}{*}{ Variable } & \multicolumn{2}{|l|}{ Left hands } & \multicolumn{2}{|l|}{ Right hands } \\
\hline & \multicolumn{2}{|l|}{ Means } & \multicolumn{2}{|l|}{ Means } \\
\hline & $\boldsymbol{R h}$ & Normal & $\boldsymbol{R h}$ & Normal \\
\hline $\begin{array}{l}\text { APD } \\
\text { AMD } \\
\text { Carpal ratio }\end{array}$ & $\begin{array}{c}10 \cdot 443 \\
88 \cdot 571^{*} \\
9 \cdot 4015^{* *}\end{array}$ & $\begin{array}{l}11 \cdot 250 \\
84 \cdot 958 \\
12 \cdot 4417\end{array}$ & $\begin{array}{l}10 \cdot 800 \\
87 \cdot 314^{* *} \\
9 \cdot 3277^{* *}\end{array}$ & $\begin{array}{l}13 \cdot 373 \\
81 \cdot 059 \\
12 \cdot 0750\end{array}$ \\
\hline
\end{tabular}

Results of the individual $t$ tests comparing the 2 groups for the mentioned variables. ${ }^{*}$ Represents significance at a $5 \%$ level. ${ }^{* *}$ Represents significance at a $1 \%$ level.

Table 2 Summary of correlation coefficients between variables

\begin{tabular}{llllll}
\hline Variable & \multicolumn{2}{l}{ Rheumatoid } & \multicolumn{2}{l}{ Normal } \\
\cline { 2 - 3 } \cline { 5 - 6 } \cline { 5 - 6 } & Left & Right & Left & Right \\
\hline $\begin{array}{l}\text { APD and AMD } \\
\begin{array}{c}\text { AMD and } \\
\text { carpal ratio }\end{array}\end{array}$ & $0.5594^{* *}$ & $0.5750^{* *}$ & -0.0357 & 0.1832 \\
$\begin{array}{c}\text { APD and } \\
\text { carpal ratio }\end{array}$ & 0.10036 & 0.2179 & 0.0309 & 0.0770 \\
\hline
\end{tabular}

** Significant at a $1 \%$ level 
NORMALS
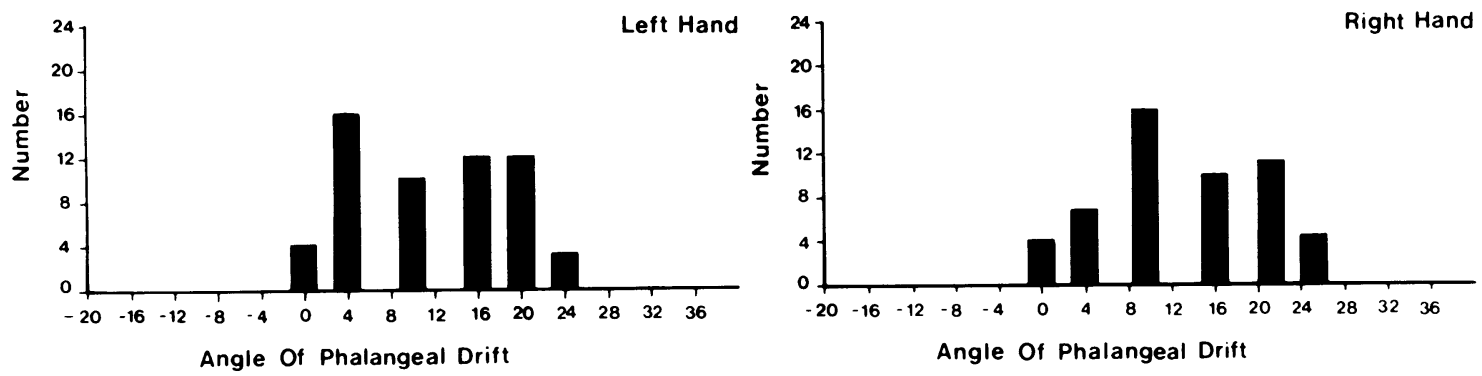

RHEUMATOIDS
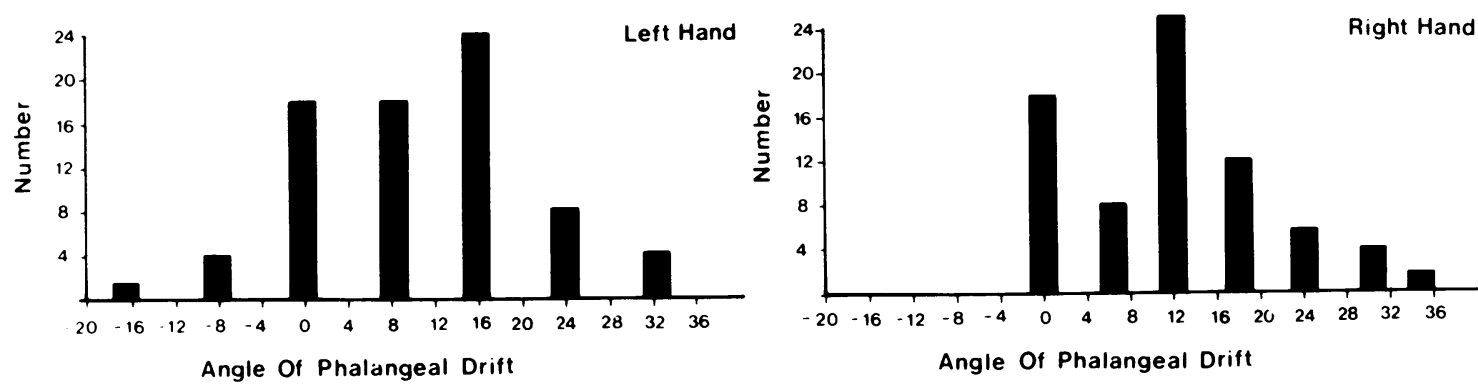

Fig. 5 Frequency distribution of angles of phalangeal drift $(A P D)$ in patients with rheumatoid arthritis and in normal controls.

In the normal hands there was no significant correlation between phalangeal deviation and metacarpal deviation.

Carpal ratio. The rheumatoid group showed marked collapse of the carpal height and a corresponding diminution of the carpal ratio. The difference from the normal was highly significant (Table 1). However, there was no significant correlation between the degree of wrist collapse and either metacarpal or the phalangeal deformity (Table 2).

\section{Discussion}

The present study was not specifically designed to establish the incidence of ulnar deviation of the fingers-the so-called classical deformity-in rheumatoid arthritis. By including patients with different stages of disease, and some who had undergone operations on the wrist or hand, we obtained a sample with a wide spectrum of deformity. Indeed, metacarpophalangeal deformity ranged from $16^{\circ}$ of radial deviation to $36^{\circ}$ of ulnar deviation, giving a mean angle which did not differ significantly from that in the normal control group.

Our principle objective was to investigate the relationship between finger deformity and wrist deformity. The results supported the hypothesis that there is a significant relationship between these 2 variables in patients with rheumatoid arthritis but not in normal persons. Given that the hands and wrists are involved in the inflammatory process, lateral deformity at one level is accompanied by a complementary opposite deformity at the other. This is consistent with the principle of an intercalated zigzag as proposed by Landsmeer ${ }^{4}$ who stated that in a biarticular system bridged by 2 biarticular muscles, the intercalated bone will take up a position in which the muscular system is shortened most, and for that reason contains the minimum for potential energy.

The question that remains unanswered is which comes first-wrist deformity or finger deformity. Examples in our own series suggest that wrist devia- 


\section{NORMALS}

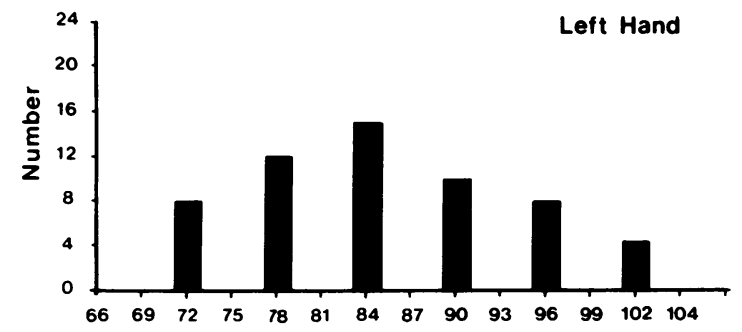

Angle of Metacarpal Deviation

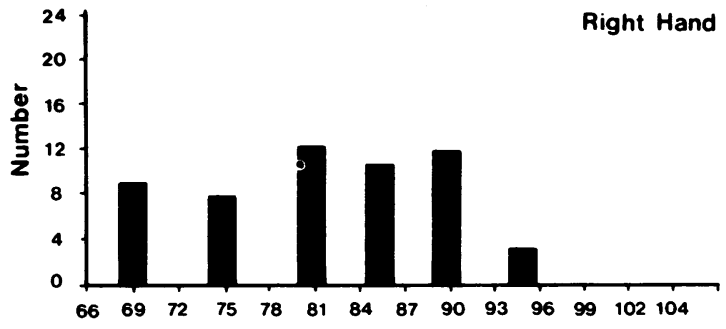

Angle Of Metacarpal Deviation

\section{RHEUMATOIDS}
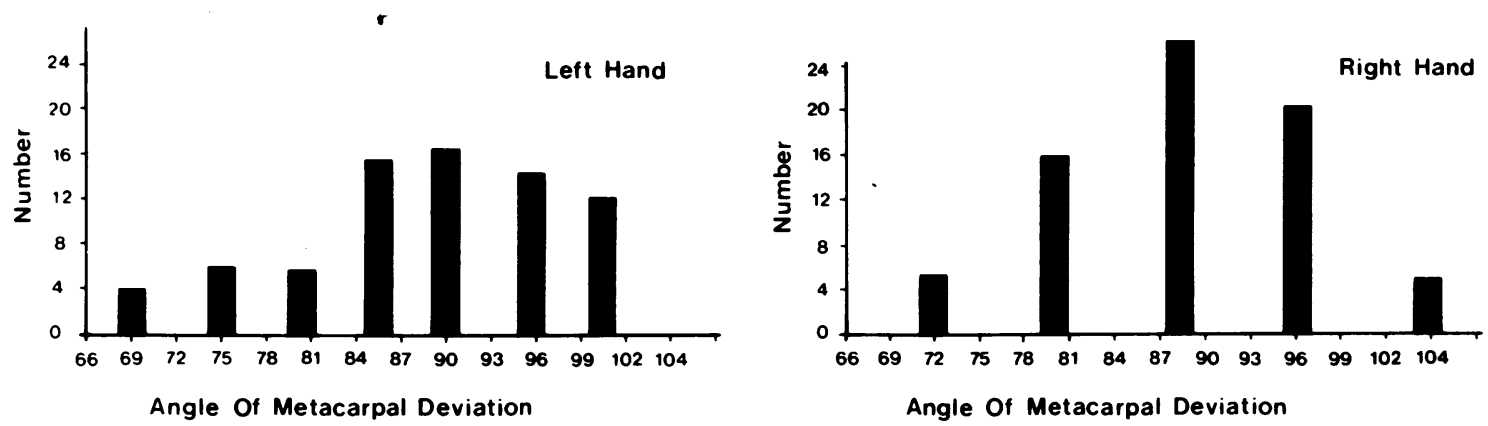

Fig. 6 Frequency distribution of angles of metacarpal deviation (AMD) in patients with rheumatoid arthritis and in normal controls.

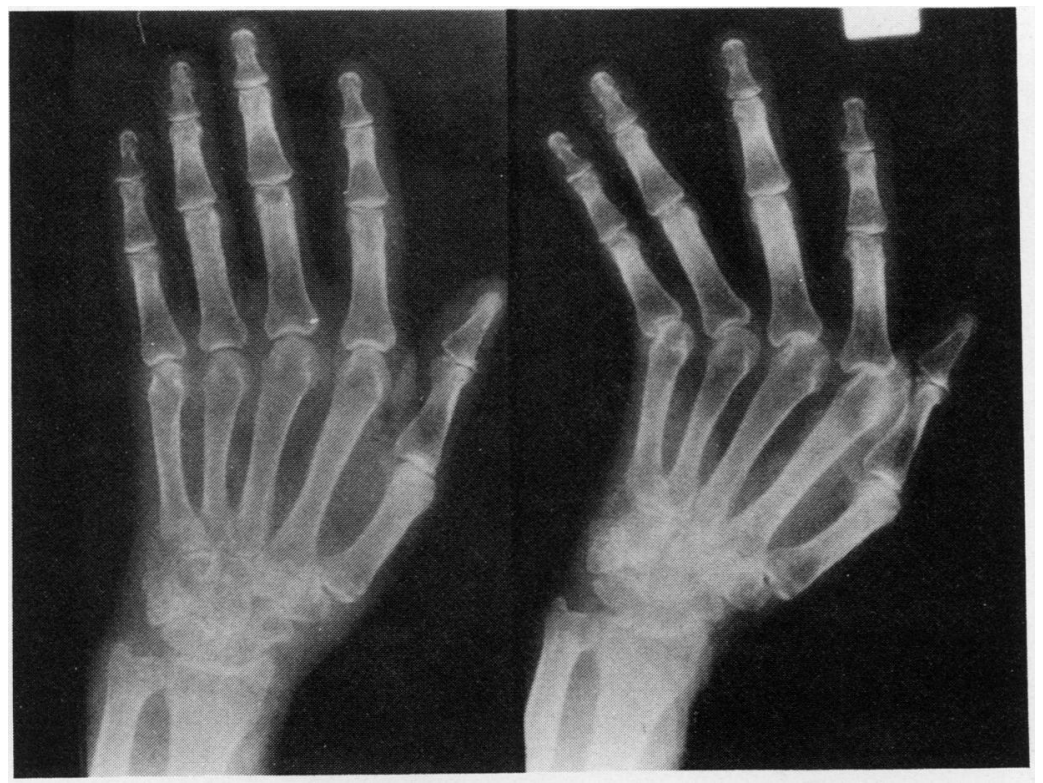

Fig. 7 Radiographs of the left hand taken 18 months apart. As radial deviation of the wrist increases so does ulnar drift of the fingers. 


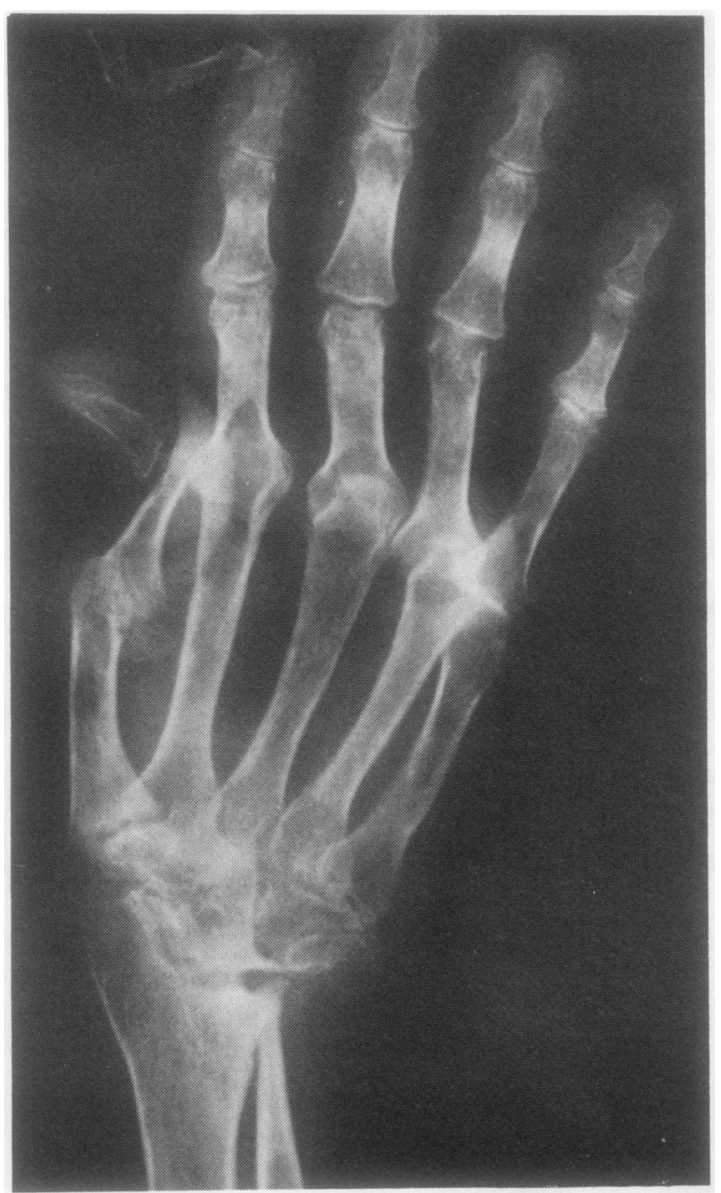

Fig. 8 Radiograph of the right hand showing that the less common radial drift of the fingers is associated with ulnar deviation of the wrist. tion precedes finger deviation (Fig. 9), and it is our contention that the former largely determines the latter.

Regardless of whether wrist deviation actually causes the complementary deformity of the fingers, it is still sound practice to correct the proximal deformity in order to prevent the progression (or recurrence) of the distal deformity. It is noteworthy that Pahle and Raunio ${ }^{5}$ found that there was a significantly lower incidence of ulnar displacement of the fingers following wrist arthrodesis in a position of ulnar deviation. In our own Arthritis Clinic we have adopted a policy of prophylactic soft-tissue correction of wrist deformity before the onset of metacarpophalangeal deviation. The extensor carpi ulnaris is explored. If it is torn or attenuated, appropriate reconstruction is carried out (if necessary, together with synovectomy) to restore continuity and normal tension. In addition, if motor imbalance can be demonstrated at the wrist, a transfer of extensor carpi radialis longus to the ulnar side is performed.

We gratefully acknowledge the assistance of Dr S. D. Reinach and Mrs Grundling, of the Institute of Biostatistics (MRC), Johannesburg.

\section{References}

1 Shapiro J S. A new factor in the aetiology of ulnar drift. Clin Orthop 1970; 68; 32-43.

2 Shapiro J S., Heijna W., Nasatir S, et al. The relationship of wrist motion to ulnar phalangeal drift in the rheumatoid patient. Hand 1971; 3: 68-75.

3 Linscheid R L, Dobyn J H. Rheumatoid arthritis of the wrist. Orthop Clin North Am 1979; 2: 649-65.

4 Landsmeer J M F. Studies in the anatomy of articulation II. Patterns of movement of bi-muscular bi-articular systems. Acta Morphol Neerl Scand. 1960; 3: 304-21.

5 Pahle J A, Raunio P. The influence of wrist position on finger deviation in the rheumatoid hand.J Bone Joint Surg 1969; 51B: 664-76. 


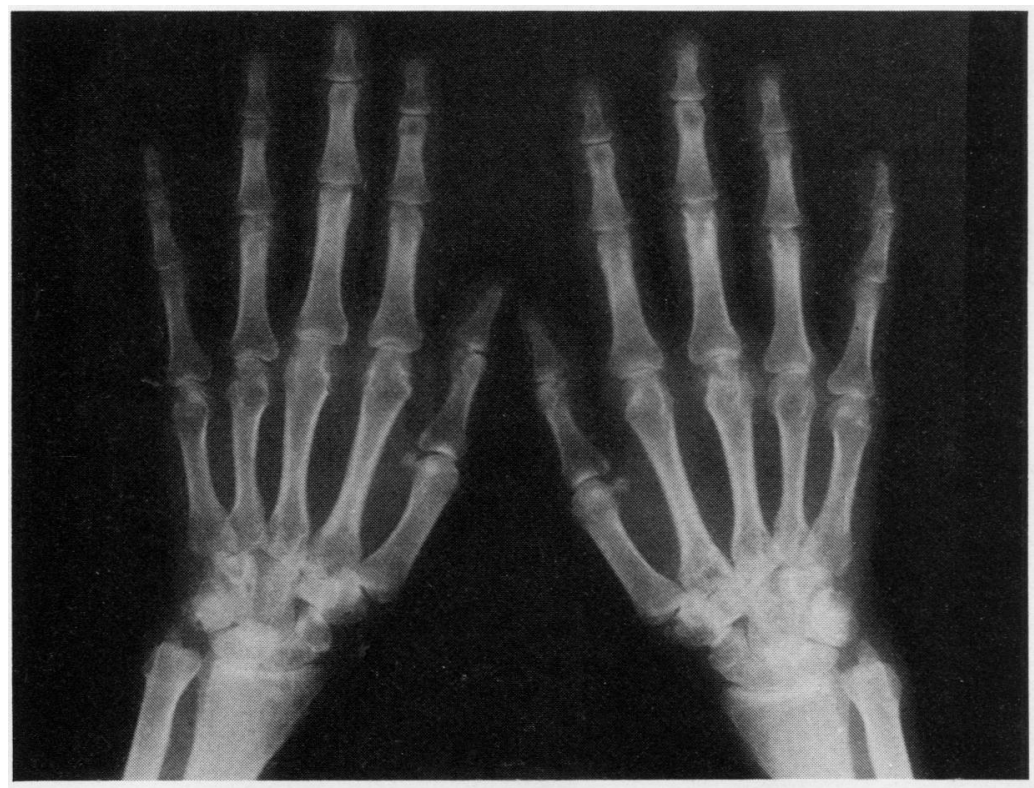

Fig. 9A

Fig. 9 Radiographs of the hands taken (A) in 1980 and (B) in 1981. Over the period of one year radial deviation of the wrist has increased on both sides, and on the left, where

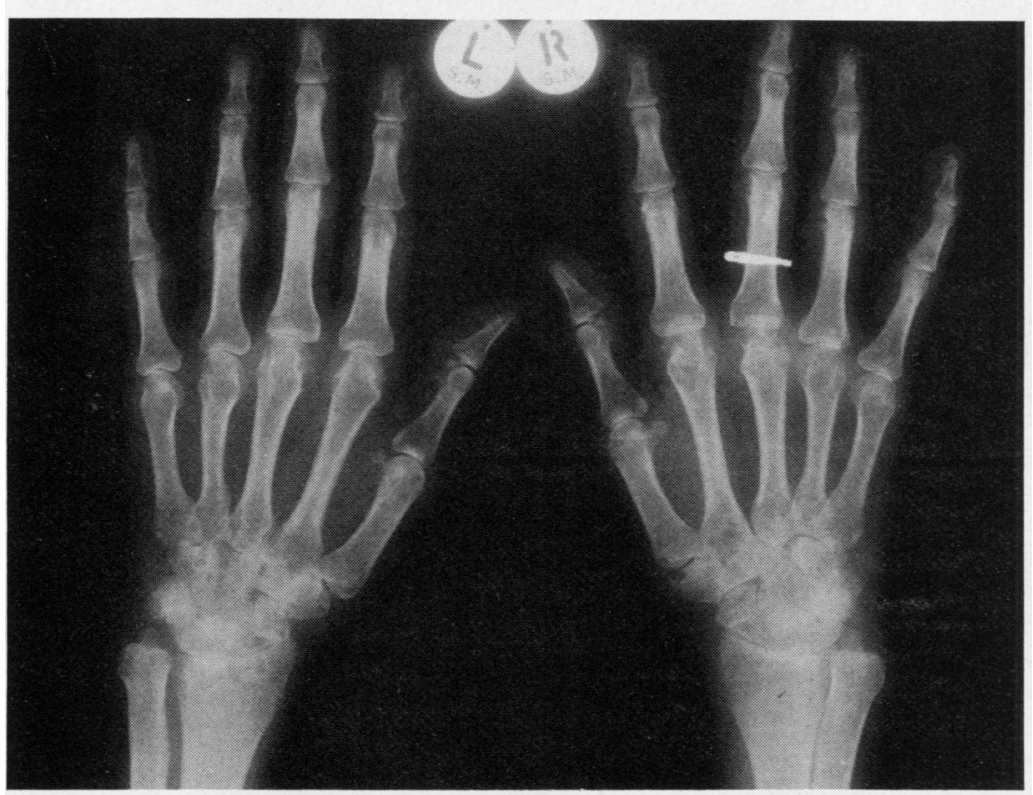
this is greatest, the fingers are beginning to drift ulnarwards.

Fig. 9B 